\title{
Adding random edges to dense graphs
}

\author{
Tom Bohman* $\quad$ Alan Frieze ${ }^{\dagger} \quad$ Michael Krivelevich $^{\ddagger} \quad$ Ryan Martin $^{\S}$
}

\begin{abstract}
This paper investigates the addition of random edges to arbitrary dense graphs; in particular, we determine the number of random edges required to ensure various monotone properties including the appearance of a fixed size clique, small diameter and $k$-connectivity.
\end{abstract}

\section{Introduction}

We consider the following random graph model, which was introduced in [1]: a fixed graph $H=(V, E)$ is given and a set $R$ of $m$ edges is chosen uniformly at random from $\left(\begin{array}{c}V \\ 2\end{array}\right) \backslash E$ (i.e. $R$ is a subset of the non-edges of $H$ ) in order to form a graph

$$
G_{H, m}=(V, E \cup R) .
$$

For a fixed constant $0<d<1$ let $\mathcal{G}(n, d)$ denote the set of graphs on vertex set $[n]$ which have minimum degree at least $d n$. We will refer to these graphs as dense graphs. We consider $G_{H, m}$ where $H$ is an arbitrary graph in $\mathcal{G}(n, d)$.

In the classical model of a random graph (Erdős and Rényi [6]) we add random edges to an empty graph, all at once or one at a time, and then ask for the probability that certain structures occur. This model is well-studied: it has generated a wide variety of research including at least two excellent texts, Bollobás [2] and Janson, Łuczak and Ruciński [8]. There are other random graph models as well. For example, there is a well established theory of considering random subgraphs of special graphs. In fact, our model can be seen to be an analogue by taking complements.

The property investigated in [1] was hamiltonicity: How large must $m$ be in order to ensure that, with high probability, $G_{H, m}$ is hamiltonian (we will say that an

\footnotetext{
*Department of Mathematical Sciences, Carnegie Mellon University, Pittsburgh, PA 15213. E-mail: tbohman@moser.math. cmu.edu. Supported in part by NSF grant DMS-0100400.

$\dagger^{\dagger}$ Department of Mathematical Sciences, Carnegie Mellon University, Pittsburgh, PA 15213. E-mail: alan@random.math.cmu.edu. Supported in part by NSF grant CCR-9818411.

${ }^{\ddagger}$ Department of Mathematics, Sackler Faculty of Exact Sciences, Tel Aviv University, Tel Aviv 69978, Israel. E-mail: krivelev@post.tau.ac.il. Research supported in part by a USA-Israel BSF grant, by a grant from the Israel Science Foundation and by a Bergmann Memorial Grant.

$\S$ Department of Mathematical Sciences, Carnegie Mellon University, Pittsburgh, PA 15213. E-mail: rymartin@andrew.cmu.edu. Supported in part by NSF VIGRE grant DMS-9819950.
} 
event occurs with high probability, abbreviated whp, if the probability of that event approaches 1 as $n \rightarrow \infty)$ ? It was proved in [1] that there exists a constant $c$, depending on $d$, such that $m \geq c n$ ensures a Hamilton cycle in $G_{H, m}$ whp. In addition, there is a constant $C$, depending on $d$, and a graph $H$ with minimum degree $d n$ which remains non-hamiltonian when adding as many as $C n$ edges. It is well-known (see [2], ch. VIII) that the threshold function for the number of random edges required to induce a Hamilton cycle in an empty graph is $\lfloor(n / 2)(\log n+\log \log n+\phi(n))\rfloor$ for any function $\phi(n) \rightarrow \infty$ as $n \rightarrow \infty$. Thus, although there are more total edges necessary to ensure the Hamilton cycle in our model than in $G_{n, M}$, we require fewer random edges.

In this paper we consider the clique number, chromatic number, diameter and vertex connectivity of $G_{H, m}$. For most of our results, we will be most interested in the case where $d$ is a small constant. In the case of a Hamilton cycle, for example, no random edges are required for $d \geq 1 / 2$. Note also that connectivity is one of the first properties to emerge when adding edges. Since every connected component in a dense graph has at least $d n$ vertices, adding $\omega(1)$ random edges will cause the resulting graph to be connected whp. Thus, if $m$ random edges give a certain monotone property monotone $P$ whp then $m+\omega(1)$ random edges edges give whp a graph that is both connected and has property $P$.

We first look at the number of random edges needed to force $G_{H, m}$ to contain a copy of $K_{r}$ where $r$ is a constant. For an arbitrary fixed graph $H$ let

$$
m(H)=\max \left\{\frac{e\left(H^{\prime}\right)}{v\left(H^{\prime}\right)}: H^{\prime} \subseteq H, v\left(H^{\prime}\right)>0\right\} .
$$

For the standard random graph we have the following.

Theorem 1 (Bollobás [3]). If $H$ is a fixed graph then

$$
\lim _{n \rightarrow \infty} \operatorname{Pr}\left(G_{n, M} \supseteq H\right)= \begin{cases}1, & \text { if } M=\omega\left(n^{2-1 / m(H)}\right) ; \\ 0, & \text { if } M=o\left(n^{2-1 / m(H)}\right) .\end{cases}
$$

The proof of the above theorem is via the second moment method. Let $X_{H}$ denote the number of copies of $H$ in $G_{n, M}$. One can conclude something slightly stronger than stated:

$$
\text { If } M=\omega\left(n^{2-1 / m(H)}\right) \text { then whp } X_{H} \rightarrow \infty \text {. }
$$

Note that $m\left(K_{r}\right)=(r-1) / 2$. Furthermore, note that if $d \geq \frac{r-2}{r-1}$ then, by the classic theorem of Turán [5], only a balanced $(r-1)$-partite graph fails to contain a complete $K_{r}$. So, we are only interested in $d<\frac{r-2}{r-1}$. Our main result here is the following.

Theorem 2. Let $r>r_{0} \geq 2$ be integers. Let $d \in\left(\frac{r_{0}-2}{r_{0}-1}, \frac{r_{0}-1}{r_{0}}\right]$ be a fixed constant. (a). If $H \in \mathcal{G}(n, d)$ and $m=\omega\left(n^{2-2 /\left(\left\lceil r / r_{0}\right\rceil-1\right)}\right)$ then $G_{H, m}$ contains a $K_{r}$ whp. 
(b). There exists a graph $H_{0} \in \mathcal{G}(n, d)$ such that if $m=o\left(n^{2-2 /\left(\left\lceil r / r_{0}\right\rceil-1\right)}\right)$, then whp $G_{H_{0}, m}$ fails to contain a $K_{r}$.

Note that if $r \leq 2 r_{0}$ then only $\omega(1)$ random edges are needed to create a $K_{r}$.

For $d \leq 1 / 2$ the minimum value of the chromatic number of $G_{H, m}$ taken over $H \in \mathcal{G}(n, d)$ is largely determined by $\chi\left(G_{n, m}\right)$ (the latter is well-studied - see, for example, [7] and [10]). This observation follows from the simple bound $\chi\left(G_{H, m}\right) \geq$ $\chi\left(G_{n, m}\right)$ and the fact that $\chi\left(G_{K_{n / 2, n}, m}\right)$ is at most twice $\chi\left(G_{n / 2, m}\right)$. In one situation Theorem 2 strengthens this lower bound: if $m=\omega(1)$ then whp $G_{H, m}$ contains a $K_{4}$ and $\chi\left(G_{H, m}\right) \geq 4$.

We now turn to the diameter of $G_{H, m}$, which we denote $\operatorname{diam}\left(G_{H, m}\right)$. If $d \geq 1 / 2$ then $\operatorname{diam}(H)=2$, and all remaining edges are required to achieve diameter 1 . We first show that only a small set of random edges is required to get $\operatorname{diam}\left(G_{H, m}\right) \leq 5$.

Theorem 3. If $H \in \mathcal{G}(n, d)$ and $m=\omega(1)$ then $\mathbf{w h p} \operatorname{diam}\left(G_{H, m}\right) \leq 5$.

Note that the number of edges required here is independent of $d$. By way of comparison, we note that Bollobás and Chung showed that whp the diameter of a graph consisting of a random matching added to an $n$-cycle is about $\log _{2} n$ [4]. Next we show that in order to get $\operatorname{diam}\left(G_{H, m}\right)<5$ roughly $\log n$ random edges are required.

\section{Theorem 4.}

(a). Let $H$ be a graph in $\mathcal{G}(n, d)$. If $m=\frac{1-d}{d^{2}} \log n+\omega(1)$ then $\mathbf{w h p} \operatorname{diam}\left(G_{H, m}\right) \leq$ 3 .

(b). If $d<1 / 2$ then there exists a graph $H_{0} \in \mathcal{G}(n, d)$ such that if $m=\frac{\log n}{-2 \log (1-2 d)}-$ $\omega(1)$ then whp $\operatorname{diam}\left(G_{H_{0}, m}\right) \geq 5$.

Finally, we prove that about $n \log n$ random edges are required to get $\operatorname{diam}(G) \leq 2$.

\section{Theorem 5.}

(a). Let $H$ be a graph in $\mathcal{G}(n, d)$. If $m=\frac{1-d}{d} n \log n+\omega(n)$ then $\mathbf{w h p} \operatorname{diam}\left(G_{H, m}\right) \leq$ 2 .

(b). There exists a graph $H_{0} \in \mathcal{G}(n, 1 / 2)$ such that if $m=\frac{1}{2} n \log n-\omega(n)$ then whp $\operatorname{diam}\left(G_{H_{0}, m}\right) \geq 3$.

Theorems 3, 4, and 5 establish intervals for $m$ in which the worst case diameter of $G_{H, m}$ is 5, 3 and 2. Note that there is, in a sense, a jump in the worst case diameter from 5 to 3 . 
The final property we consider is vertex connectivity. First note that if $d>1 / 2$ then $H \in \mathcal{G}(n, d)$ is at least $(2\lceil d n\rceil-n+2)$-connected. This can be seen by the fact that, by removing any set of size $2\lceil d n\rceil-n$, the resulting graph has minimum degree at least half the number of vertices. This means that the resulting graph has a Hamilton cycle and is, thus, at least 2-connected. Since there are graphs $H$ which are disconnected but have minimum degree $d n(d<1 / 2)$, we focus on the number of random edges required to make $G_{H, m} k$-connected for $k \leq c n$, for some constant $c=c(d)>0$.

\section{Theorem 6.}

(a). Let $H \in \mathcal{G}(n, d)$. If $k=O(1)$ and $m=\omega(1)$ then $G_{H, m}$ is $k$-connected, whp. If $\omega(1) \leq k \leq d^{2} n / 32$ and $m=640 k / d^{2}$ then $G_{H, m}$ is $k$-connected, whp.

(b). If $d<1 / 2$ then there exists an $H_{0} \in \mathcal{G}(n, d)$ such that whp $G_{H_{0}, m}$ fails to be $k$-connected for all $k$ such that $m \leq \frac{k}{2}\left\lfloor\frac{n}{d n+1}\right\rfloor$.

The remainder of the paper is organized as follows. In the next section we establish some preliminaries that will be used in the proofs. Theorem 2 is proved in Section 3. In Section 4 we prove Theorems 3, 4 and 5. Finally, we prove Theorem 6 in Section 5.

Remark 1. All of the properties considered here are monotone increasing. We therefore have an alternative way of adding random edges. We can take each nonedge of $H$ and add it with probability $p$, creating the random graph $G_{H, p}$ where $\left(\left(\begin{array}{l}n \\ 2\end{array}\right)-|E(H)|\right) p \leq m$. It then follows, as in Bollobás [2], Theorem 2.2, that for a monotone increasing property $\mathcal{P}, G_{H, p} \in \mathcal{P}$ whp implies $G_{H, m} \in \mathcal{P}$ whp. This observation can sometimes simplify our calculations.

\section{Preliminaries}

We will use the powerful Regularity Lemma of Szemerédi, which was formally introduced in [11]. A survey of applications of the Regularity Lemma is given in in [9]. In order to state the Lemma, we need a definition. For two disjoint vertex sets $A$ and $B$, let $e(A, B)$ denote the number of edges with one vertex in $A$ and the other in $B$. Also, let $d(A, B)=\frac{e(A, B)}{|A||B|}$.

Definition 1 (Regularity condition). Let $\epsilon>0$. Given a graph $G$ and two disjoint vertex sets $A \subset V, B \subset V$, we say that the pair $(A, B)$ is $\epsilon$-regular if for every $X \subset A$ and $Y \subset B$ satisfying

$$
|X|>\epsilon|A| \quad \text { and } \quad|Y|>\epsilon|B|
$$

we have

$$
|d(X, Y)-d(A, B)|<\epsilon .
$$


Now we can state the Regularity Lemma itself. We will not need the classical form but instead a modification, known as the Degree Form.

Theorem 7 (Regularity Lemma, Degree Form). For every $\epsilon>0$ there is an $M=M(\epsilon)$ such that if $G=(V, E)$ is any graph and $\delta \in[0,1]$ is any real number, then there is a partition of the vertex-set $V$ into $\ell+1$ clusters $V_{0}, V_{1}, \ldots, V_{\ell}$, and there is a spanning subgraph $G^{\prime} \subset G$ with the following properties:

- $\ell \leq M$

- $\left|V_{0}\right| \leq \epsilon|V|$,

- all clusters $V_{i}, i \geq 1$, are of the same size $L \leq\lceil\epsilon|V|\rceil$,

- $\operatorname{deg}_{G^{\prime}}(v)>\operatorname{deg}_{G}(v)-(\delta+\epsilon)|V|$, for all $v \in V$,

- $e\left(G^{\prime}\left[V_{i}\right]\right)=0$, for all $i \geq 1$,

- all pairs $G^{\prime}\left(V_{i}, V_{j}\right),(1 \leq i<j \leq \ell)$ are $\epsilon$-regular, each with a density either 0 or greater than $\delta$.

The sets $V_{i}(1 \leq i \leq \ell)$ are defined to be clusters. In addition, we define the reduced graph. This is the graph, usually denoted $G_{r}$, which has $\left\{V_{1}, \ldots, V_{\ell}\right\}$ as its vertex set and $V_{i} \sim_{G_{r}} V_{j}$ if and only if the pair $\left(V_{i}, V_{j}\right)$ is $\epsilon$-regular with density at least $\delta$ in $G^{\prime}$.

In papers utilizing the Regularity Lemma, it is customary to use the notation "«". The expression $\epsilon \ll \delta$ means that $\epsilon$ is "small enough, relative" to $\delta$.

As we have discussed, all the properties we consider are monotone; that is, if $G$ has the property in question, then all graphs that have $G$ as a subgraph will have the property as well. At several times throughout this paper, we will want to add edges to a subgraph $H^{\prime}$ instead of the original graph $H$; often, the subgraph given by Theorem 7 . Let $\mathcal{P}$ be a monotone property of graphs. A simple coupling argument gives the intuitive result:

Proposition 1. Let $H^{\prime} \subset H$. Let $G^{\prime}$ be the graph formed by adding $m$ random edges to $H^{\prime}$ and $G$ be the graph formed by adding $m$ random edges to $H$. Then

$$
\operatorname{Pr}\left\{G^{\prime} \text { has property } \mathcal{P}\right\} \leq \operatorname{Pr}\{G \text { has property } \mathcal{P}\} \text {. }
$$

\section{Complete subgraphs}

Proof of Theorem 2. Let us first prove (b). Let $H_{0}$ be a complete $r_{0}$-partite graph with nearly equal parts. Note that if $r \leq 2 r_{0}$ then we are adding no random edges, 
so we henceforth assume $r>2 r_{0}$. Let $A_{1}, \ldots, A_{r_{0}}$ be the parts of the $r_{0}$-partition of $V\left(H_{0}\right)$. By the choice of $m$ and Theorem 1, whp the induced graph $G\left[A_{i}\right]$ contains no $K_{\left\lceil r / r_{0}\right\rceil}$ for $i=1, \ldots, r_{0}$. If this is the case, then the largest clique in $G$ has size

$$
r_{0}\left(\left\lceil r / r_{0}\right\rceil-1\right)<r
$$

To prove part (a) we apply the degree form of the Regularity Lemma. Choose $\epsilon$ and $\delta$ so that $\epsilon \ll \delta \ll d, 1 / r$ and apply Theorem 7 to $H$ with those values, giving us the subgraph $H^{\prime}$. The reduced graph has minimum degree

$$
(d-\delta-2 \epsilon) \ell>\frac{r_{0}-2}{r_{0}-1} \ell
$$

for $\delta, \epsilon$ sufficiently small. Therefore, it follows from Turán's theorem that there is a $K_{r_{0}}$ in the reduced graph. In other words, there exists a collection of clusters $A_{1}, \ldots, A_{r_{0}} \subseteq V\left(H^{\prime}\right)$ such that each pair of these sets gives an $\epsilon$-regular pair with density at least $\delta$.

We use a fact from [9]. For an arbitrary graph $H$ and $v \in V(H)$ let $N(v)$ be the set of vertices adjacent to $v$ in $H$.

Proposition 2 (Intersection Property). Let $(A, B)$ be an $\epsilon$-regular pair with density $\delta$. If $Y \subseteq B$, and $(\delta-\epsilon)^{k-1}|Y|>\epsilon|B|$ then

$$
\#\left\{\left(x_{1}, x_{2}, \ldots, x_{k}\right): x_{i} \in A\left|\left(\bigcap_{i=1}^{k} N\left(x_{i}\right)\right) \cap Y\right| \leq(\delta-\epsilon)^{k}|Y|\right\} \leq k \epsilon|A|^{k}
$$

We add $\omega\left(n^{2-2 /\left(\left\lceil r / r_{0}\right\rceil-1\right)}\right)$ random edges to $H^{\prime}$ in a sequence of $r_{0}$ phases. $\varphi=\varphi(n)$ will be a function that goes to infinity as $n \rightarrow \infty$ and we let $R=R_{1} \cup \cdots \cup R_{r_{0}}$ where $\left|R_{i}\right|=\varphi^{2} n^{2-2 /\left(\left\lceil r / r_{0}\right\rceil-1\right)}$ for $i=1, \ldots, r_{0}$. (Recall that $R$ is the set of random edges.)

It follows from (1) that whp $R_{1} \cap\left(\begin{array}{c}A_{1} \\ 2\end{array}\right)$ contains at least $\varphi$ copies of $K_{\left\lceil r / r_{0}\right\rceil}$. It follows from the Intersection Property that among these whp there exists a copy of $K_{\left\lceil r / r_{0}\right\rceil}$ such that intersection of the neighborhoods of the vertices in this $K_{\left\lceil r / r_{0}\right\rceil}$ is large in $A_{2}, \ldots, A_{r_{0}}$; to be precise, whp there exists $X_{1} \subseteq A_{1}$ such that

(i) $\left|X_{1}\right|=\left\lceil r / r_{0}\right\rceil$,

(ii) $\left(\begin{array}{c}X_{1} \\ 2\end{array}\right) \subseteq R_{1}$, and

(iii) For $i=2, \ldots, r_{0}$ we have

$$
\left|\left(\bigcap_{x \in X_{1}} N(x)\right) \cap A_{i}\right| \geq(\delta-\epsilon)^{\left\lceil r / r_{0}\right\rceil}\left|A_{i}\right|
$$


For $i=2, \ldots, r_{0}$ let

$$
A_{i, 1}=\left(\bigcap_{x \in X_{1}} N(x)\right) \cap A_{i}
$$

In the phase $j, j=2, \ldots, r_{0}$, we note that it follows from Theorem 1 that whp $R_{j} \cap\left(\begin{array}{c}A_{j, j-1} \\ 2\end{array}\right)$ contains at least $\varphi$ copies of $K_{\left\lceil r / r_{0}\right\rceil}$. It then follows from an application of the Intersection Property that whp there exists $X_{j} \subseteq A_{j, j-1}$ such that

(i) $\left|X_{j}\right|=\left\lceil r / r_{0}\right\rceil$,

(ii) $\left(\begin{array}{c}X_{j} \\ 2\end{array}\right) \subseteq R_{j}$, and

(iii) For $i=j+1, \ldots, r_{0}$ we have

$$
\left|\left(\bigcap_{x \in X_{j}} N(x)\right) \cap A_{i, j-1}\right| \geq(\delta-\epsilon)^{\left\lceil r / r_{0}\right\rceil}\left|A_{i, j-1}\right|
$$

If $j<r_{0}$ then, for $i=j+1, \ldots, r_{0}$, let

$$
A_{i, j}=\left(\bigcap_{x \in X_{j}} N(x)\right) \cap A_{i, j-1} .
$$

If $j=r_{0}$ then note that $X_{1} \cup \cdots \cup X_{r_{0}}$ is a $K_{r}$ in $G_{H, m}$.

Note that this argument requires

$$
\frac{k \epsilon}{(\delta-\epsilon)^{r_{0} k^{2}}}<1 \quad \text { and } \quad(\delta-\epsilon)^{r_{0} k}>\epsilon
$$

where $k=\left\lceil r / r_{0}\right\rceil$. Of course, we can attain these inequalities by choosing $\epsilon$ sufficiently small.

Remark 2. The proof of Theorem 2 (a) goes through if the condition $H \in \mathcal{G}(n, d)$ is replaced with the condition

$$
\sum_{v \in V(H)} \operatorname{deg}(v) \geq 2 d|V(H)|
$$

i.e. the average degree of $H$ is at least d. Just as a minimum degree condition on $H$ implies a minimum degree condition on $H^{\prime}$ that in turns implies a minimum degree condition on the reduced graph, a lower bound on the average degree of $H$ implies a lower bound on the average degree of $H^{\prime}$ that in turn gives a lower bound on the average degree of the reduced graph. Such a bound on the average degree is all that is required to establish the existence of a $K_{\left\lceil r / r_{0}\right\rceil}$ in the reduced graph (by Turán's Theorem). 


\section{Diameter}

Proof of Theorem 3. We apply the Regularity Lemma to $H$ with parameters $\epsilon \ll$ $\delta \ll d$. This gives us the reduced graph $H_{r}$ as well as the subgraph $H^{\prime}$. We will work with $H^{\prime}$ instead of $H$ by invoking Proposition 1.

For each cluster $V$ let $V^{\prime}$ be a fixed cluster such that $\left(V, V^{\prime}\right)$ is, in $H^{\prime}, \epsilon$-regular with density at least $\delta$ (i.e. $V \sim V^{\prime}$ in $H_{r}$ ). Note that for every vertex $x$ there exists a cluster $V_{x}$ in which $x$ has large degree in $H^{\prime}$ (note that $x \notin V_{x}$ ). Indeed, using the conditions of Theorem 7 , the minimum degree condition and the fact that $\left|V_{0}\right| \leq \epsilon n$, we see that $x$ is adjacent to at least $d n-(\delta+\epsilon) n-\epsilon n=(d-\delta-2 \epsilon) n$ vertices in $\bigcup_{i=1}^{\ell} V_{i}$. So, there is a cluster $V_{x}$ such that $x$ is adjacent to at least

$$
\frac{(d-\delta-2 \epsilon) n}{\ell} \geq(d-\delta-2 \epsilon) L
$$

vertices in $V_{x}$.

For arbitrary vertices $u$ and $w$ we find a path of length at most 5 from $u$ to $w$ using the clusters $V_{u}, V_{w}, V_{u}^{\prime}$ and $V_{w}^{\prime}$. Since $V_{u} \sim_{H_{r}} V_{u}^{\prime}$, at least $(1-\epsilon) L$ vertices in $V_{u}^{\prime}$ are at a distance two from $u$. The same holds for $w$. It follows, for example, that $V_{u}^{\prime}=V_{w}^{\prime}$ implies $\operatorname{dist}(u, w) \leq 4$. It is also easy to see that if $V_{u}^{\prime} \sim_{H_{r}} V_{w}^{\prime}$ then $\operatorname{dist}(u, w) \leq 5$. So, we may assume that the clusters $V_{u}, V_{w}, V_{u}^{\prime}$ and $V_{w}^{\prime}$ are distinct and

$$
V_{u} \sim_{H_{r}} V_{u}^{\prime} \quad V_{u}^{\prime} \nsim_{H_{r}} V_{w}^{\prime} \quad V_{w}^{\prime} \sim_{H_{r}} V_{w} .
$$

We use the random edges between $V_{u}^{\prime}$ and $V_{w}^{\prime}$ to complete the path from $u$ to $w$.

For a fixed constant $k$ and fixed clusters $V_{1}, V_{2}, V_{3}, V_{4}$ such that

$$
V_{1} \sim_{H_{r}} V_{2} \quad \text { and } \quad V_{3} \sim_{H_{r}} V_{4}
$$

we define a $k$-link of the quadruple $V_{1}, V_{2}, V_{3}, V_{4}$ to be a collection of $k^{2}$ edges

$$
\left\{e_{i, j}=\left\{a_{i, j}, b_{i, j}\right\}: 1 \leq i, j \leq k\right\}
$$

such that

(i) $a_{i, j} \in V_{2}$ for $1 \leq i, j \leq k$,

(ii) $b_{i, j} \in V_{3}$ for $1 \leq i, j \leq k$,

(iii) For $i=1, \ldots, k$ we have

$$
\left|\bigcup_{j=1}^{k} N\left(a_{i, j}\right) \cap V_{1}\right|>L-(d-\delta-2 \epsilon) L
$$

(iv) For every function $\sigma:[k] \rightarrow[k]$ we have

$$
\left|\bigcup_{i=1}^{k} N\left(b_{i, \sigma(i)}\right) \cap V_{4}\right|>L-(d-\delta-2 \epsilon) L
$$


We show below that there exists a $k$ such that whp $R$ contains a $k$-link for every quadruple of clusters satisfying (3).

Before proving the existence of these $k$-links, we show that if $R$ contains a $k$ link of $V_{u}, V_{u}^{\prime}, V_{w}^{\prime}, V_{w}$ then there is a path of length 5 from $u$ to $v$ in $G_{H, m}$. Let $X=N(u) \cap V_{u}$ and $Y=N(w) \cap V_{w}$. It follows from (2) and (iii) that for $i=1, \ldots k$ there exists $\sigma(i) \in[k]$ such that $a_{i, \sigma(i)}$ has a neighbor $x_{i} \in X$. It then follows from (2) and (iv) that there exists $j \in[k]$ such that $b_{j, \sigma(j)}$ has a neighbor $y \in Y$. The following sequence of vertices is a path in $G_{H, m}: u, x_{j}, a_{j, \sigma(j)}, b_{j, \sigma(j)}, y, w$.

It remains to show that there exists a $k$ such that whp $R$ contains a $k$-link for every quadruple of clusters satisfying (3). Since the number of vertices in the reduced graph $H_{r}$ is bounded, it suffices to show that there exists a constant $k$ such that whp a fixed quadruple $V_{1}, V_{2}, V_{3}, V_{4}$ satisfying (3) has a $k$-link. We use the following simple fact regarding $\epsilon$-regular pairs.

Proposition 3 (Union Property). Let $(A, B)$ be an $\epsilon$-regular pair with density at least $\delta$. If $k \geq 1$ and $(1-\delta+\epsilon)^{k-1} \geq \epsilon$ then

$$
\#\left\{\left(x_{1}, x_{2}, \ldots, x_{k}\right): x_{i} \in A,\left|\bigcup_{i=1}^{k} N\left(x_{i}\right)\right| \leq\left(1-(1-\delta+\epsilon)^{k}\right)|B|\right\} \leq k \epsilon|A|^{k}
$$

Proof. We go by induction on $k$. For $k=1$ this statement follows directly from the definition of $\epsilon$-regularity. For $k \geq 2$, let $\mathcal{X}$ be the collection of $(k-1)$-tuples $x_{1}, \ldots, x_{k-1}$ of elements of $A$ with the property that

$$
\left|\bigcup_{j=1}^{k-1} N\left(x_{j}\right)\right| \leq\left(1-(1-\delta+\epsilon)^{k-1}\right)|B| .
$$

By induction, $|\mathcal{X}| \leq(k-1) \epsilon|A|^{k-1}$. For a fixed $(k-1)$-tuple $v_{1}, \ldots, v_{k-1}$ not in $\mathcal{X}$, the number of vertices that are adjacent to fewer than $(\delta-\epsilon)(1-\delta+\epsilon)^{k-1}|B|$ vertices among a set of $(1-\delta+\epsilon)^{k-1}|B|$ vertices that contain the complement of that union is at most $\epsilon|A|$, by the definition of $\epsilon$-regularity. Thus, the number of bad $k$-tuples is at most

$$
|\mathcal{X}| \cdot|A|+|A|^{k-1} \cdot \epsilon|A| \leq(k-1) \epsilon|A|^{k-1} \cdot|A|+|A|^{k-1} \cdot \epsilon|A|=k \epsilon|A|^{k} .
$$

Let $k$ be a constant such that

$$
\begin{gathered}
1-(1-\delta+\epsilon)^{k} \geq 1-(d-\delta-2 \epsilon) \\
(1-\delta+\epsilon)^{k-1} \geq \epsilon, \quad \text { and } \\
\left(k+k^{k}\right) k \epsilon<1 .
\end{gathered}
$$


Clearly, such a $k$ exists if we choose the constants $\epsilon$ and $\delta$ appropriately.

We are now ready to consider the random edges. Clearly, the probability that there are fewer than, say, $\sqrt{m}$ edges $e \in R$ that intersect both $V_{2}$ and $V_{3}$ is $o(1)$. Conditioning on this event, we may assume that there is a collection of $\sqrt{m}=\omega(1)$ edges $R^{\prime}$ between clusters $V_{2}$ and $V_{3}$ chosen uniformly at random from the collection of all such edges. It follows from the Union Property that the probability that a fixed set of $k^{2}$ such edges does not form a $k$-link is at most $\left(k+k^{k}\right) k \epsilon$. By our choice of $k, \epsilon$ and $\delta$, this probability is less than 1. Partitioning $R^{\prime}$ into $\omega(1)$ collections of $k^{2}$ edges, we see that whp one of these parts forms a $k$-link.

Proof of Theorem 4. The proof of (a) is a straightforward computation showing that every pair of disjoint neighborhoods must have an edge between them. Following Remark 1 we consider $G_{H, p}$ with $p=\frac{2(\log n+\omega(1))}{d^{2} n^{2}}$.

$$
\begin{aligned}
\operatorname{Pr}\left\{\exists u, v \in\left(\begin{array}{c}
{[n]} \\
2
\end{array}\right): R \cap(N(u) \times N(v))=\emptyset\right. \text { and } & N(u) \cap N(v)=\emptyset\} \\
\leq & \left(\begin{array}{c}
n \\
2
\end{array}\right)(1-p)^{d^{2} n^{2}} \\
& \leq\left(\begin{array}{c}
n \\
2
\end{array}\right) e^{-d^{2} n^{2} p} \\
& =o(1) .
\end{aligned}
$$

In order to prove the existence of the graph $H_{0}$ for (b) we consider the graph $H$ which is the disjoint union of $G_{\lfloor n / 2\rfloor, p}$ and $G_{[n / 2\rceil, p}$ where $p=2 d+n^{-1 / 3}$. In other words, we form a partition $[n]=S \cup T$ where $|S|=\lfloor n / 2\rfloor$ and $|T|=\lceil n / 2\rceil$, place no edges between the two parts, and place each edge that lies within one of the parts, independently, with probability $p$.

Let $R$ be an arbitrary set of $m$ edges on vertex set $[n]=S \cup T$. Let $S^{\prime}$ and $T^{\prime}$ be the vertices in $S$ and $T$, respectively, that are in edges in $R$. We begin by showing that for this fixed set $R$ whp the random graph $H$ has the following property: There exist $u \in S$ and $v \in T$ such that $(\{u\} \cup N(u)) \cap S^{\prime}=\emptyset$ and $(\{v\} \cup N(v)) \cap T^{\prime}=\emptyset$. Let $r=\left|S^{\prime}\right|$. The probability that $N(u) \cap S^{\prime}$ is nonempty for every vertex in $S \backslash S^{\prime}$ is at most

$$
\begin{aligned}
\left(1-(1-p)^{r}\right)^{n / 2-r} & =\left(1-e^{r \log (1-p)}\right)^{n / 2-r} \\
& \leq \exp \left\{-e^{r \log (1-p)}(n / 2-r)\right\} \\
& \leq \exp \left\{-e^{2 m \log (1-p)}(n / 3)\right\} \\
& =o(1) .
\end{aligned}
$$

It follows from this observation (and an application of the Chernoff bound to the degrees in $H$ ) that there exists a fixed graph $H$ such that 
(i) The minimum degree of $H$ is greater than $d n$.

(ii) whp (with respect to the random choice of $R$, now) there exist $u \in S$ and $v \in T$ such that no vertex in the set $\{u, v\} \cup N(u) \cup N(v)$ intersects an edge in $R$.

The distance in $G_{H, m}$ between the vertices $u$ and $v$ given by (ii) is at least 5 .

Proof of Theorem 5. The proof of (a) is also a straightforward calculation. We show that there is an edge between every vertex and neighborhood. Following Remark 1, we consider $G_{H, p}$ with $p=\frac{2(\log n+\omega(1))}{d n}$.

$$
\begin{aligned}
\operatorname{Pr}\{\exists u, v: R \cap(\{u\} \times N(v))=\emptyset\} & \leq\left(\begin{array}{l}
n \\
2
\end{array}\right)(1-p)^{d n} \\
& \leq\left(\begin{array}{l}
n \\
2
\end{array}\right) e^{-n p} \\
& =o(1) .
\end{aligned}
$$

The example of $H_{0}$ that we use for (b) is simply two complete graphs, each on $n / 2$ vertices, with no edges between them. Let the vertex sets for these complete graphs be $S$ and $T$. Note that if there exists a vertex $u \in S$ that is in no edge in $R$ and there exists a vertex $v \in T$ that is in no edge in $R$ then the distance between $u$ and $v$ in $G_{H_{0}, m}$ is at least 3 .

We compute the probability that $R$ touches every vertex in $S$.

$$
\begin{aligned}
\operatorname{Pr}\{\forall v \in S \exists e \in R \text { such that } v \in e\} & \leq\left(1-(1-2 / n)^{m}\right)^{n / 2} \\
& \leq\left(1-e^{-2 m / n-4 m / n^{2}}\right)^{n / 2} \\
& \leq \exp \left\{-\frac{n}{2} e^{-2 m / n-4 m / n^{2}}\right\} \\
& =o(1) .
\end{aligned}
$$

Note that we used the fact that the event $\{\exists e \in R$ such that $v \in e\}$ is negatively correlated with events of the form $\{\forall w \in W \exists e \in R$ such that $w \in e\}$, where $W \subseteq$ $S \backslash\{v\}$.

Remark 3. Improvements in the constants in Theorems 4(b) and 5(b) could be obtained by noting that a significant portion of the random edges in $R$ fall within the two parts of the partition (of course, one would have to replace the complete graphs used in the proof of Theorem 5(b) with arbitrary graphs of the desired density to get this improvement there). This observation was not used here for the sake of brevity. 


\section{$5 \quad$ Vertex connectivity}

Let $\kappa(G)$ denote the vertex connectivity of graph $G$. We first prove the following lemma that may be of independent interest.

Lemma 1. Let $H=(V, E)$ be a graph on $n$ vertices with minimum degree $k>0$. Then there exists a partition $V=V_{1} \cup \ldots \cup V_{t}$ such that for every $1 \leq i \leq t$ the set $V_{i}$ has at least $k / 8$ vertices and the induced subgraph $H\left[V_{i}\right]$ is $k^{2} /(16 n)$-connected.

Proof. Recall the following classical result on vertex connectivity.

Theorem 8 (Mader (see [5])). Every graph of average degree at least $k$ has a $k / 4$-connected subgraph.

Let $\left(C_{1}, \ldots, C_{t}\right)$ be a family of disjoint subsets of $V$ with the property that each induced subgraph $H\left[C_{i}\right]$ is $k / 8$-connected and that, among all such families of subsets, the set of vertices

$$
C \stackrel{\text { def }}{=} \bigcup_{i=1}^{t} C_{i}
$$

is maximal. According to Theorem $8, t>0$. Also, $\left|C_{i}\right| \geq k / 8$ for all $i$ and thus $t \leq 8 n / k$.

Let now $\left(V_{1}, \ldots, V_{t}\right)$ be a family of disjoint subsets of $V$ such that $C_{i} \subseteq V_{i}$, the induced subgraph $H\left[V_{i}\right]$ is $k^{2} /(16 n)$-connected for all $1 \leq i \leq t$ and that among all such families the set of vertices

$$
U \stackrel{\text { def }}{=} \bigcup_{i=1}^{t} V_{i}
$$

is maximal. We claim that $U=V$. Assume to the contrary that there exists a vertex $v \in V \backslash U$. If $\left|N(v) \cap V_{i}\right| \geq k^{2} /(16 n)$ for some $i$, then adding $v$ to $V_{i}$ can be easily seen to keep $H\left[V_{i}\right] k^{2} /(16 n)$-connected, contradicting the maximality of $U$. Thus $v$ has less than $k^{2} /(16 n)$ neighbors in each of the $t \leq 8 n / k$ sets $V_{i}$, and therefore $\operatorname{deg}_{V \backslash U} v>k-(8 n / k)\left(k^{2} /(16 n)\right)=k / 2$. We conclude that the minimum degree of the induced subgraph $H[V \backslash U]$ is at least $k / 2$. Applying Theorem 8, this time to $H[V \backslash U]$, unveils a $k / 8$-connected subgraph disjoint from $C$ - a contradiction to the choice of $\left(C_{1}, \ldots, C_{t}\right)$. Hence the family $\left(V_{1}, \ldots, V_{t}\right)$ covers indeed all the vertices of $H$ and thus forms a required partition.

We remark that the above result is optimal up to constant multiplicative factors. To see this take $\left\lceil\left(n-k^{2} / n\right) /(k+1)\right\rceil$ disjoint cliques $C_{i}$ of size $k+1$ each, add an independent set $I$ on the (at most $k^{2} / n$ ) remaining vertices, and connect each vertex of $I$ with roughly $k^{2} / n$ arbitrarily chosen vertices of $C_{i}, 1 \leq i \leq\lceil(n-$ $\left.\left.k^{2} / n\right) /(k+1)\right\rceil$. Denote the obtained graph by $H$. Let $K \subseteq H$ be a subgraph of $H$ containing some vertices from $I$. If $K$ intersects two distinct cliques $C_{i}, C_{j}$, 
then deleting $V(K) \cap I$ disconnects $V(K) \cap C_{i}$ from $V(K) \cap C_{j}$, and thus the connectivity of $K$ does not exceed $|V(K) \cap I| \leq|I| \leq k^{2} / n$. If $K$ intersects a unique clique $C_{i}$, then deleting all neighbors of $v \in V(K) \cap I$ from $C_{i}$ disconnects $v$ from the rest of $K$, implying $\kappa(H[K]) \leq \operatorname{deg}_{C_{i}} v \leq k^{2} / n$.

Proof of Theorem 6. Let us begin with part (a). Let $H$ be a graph with minimum degree at least $d n$. Let $\left(V_{1}, \ldots, V_{t}\right)$ be a partition of $V(H)$ such that $\left|V_{i}\right| \geq d n / 8$ and $H\left[V_{i}\right]$ is $\left(d^{2} n / 16\right)$-connected, $1 \leq i \leq t$. The existence of such a partition is guaranteed by Lemma 1. It is enough to show that the graph $G_{H, m}$ whp contains a matching of size $k$ in each bipartite graph induced by $\left(V_{i}, V_{j}\right)$. Let $F_{i j}$ be a maximum matching between $V_{i}$ and $V_{j}$ in $H$. If $\left|F_{i j}\right| \geq k$ we are done. Assume therefore that $\left|F_{i j}\right|<k$. Choose a subset $A \subset V_{i} \backslash \bigcup_{e \in F_{i j}} e$ and a subset $B \subset V_{j} \backslash \bigcup_{e \in F_{i j}} e$ of cardinalities $|A|=|B|=3 d n / 32$. Obviously $H$ has no edges connecting $A$ and $B$ due to the maximality of $F_{i j}$.

Consider first the case $k=O(1)$. Then the set $R$ contains whp $\omega(1)$ random edges between $A$ and $B$. If $\omega(1)=o(\sqrt{n})$, then those random edges form whp a matching as required.

Let now $k=\omega(1)$. Due to Remark 1 we may consider $G_{H, p}$ with $p=\frac{1280 k}{d^{2} n^{2}}$. Then the probability that the set $R$ of random edges does not have a matching of size $k$ between $A$ and $B$ can be estimated from above by:

$$
\sum_{i=0}^{k-1}\left(\begin{array}{c}
\frac{3 d n}{32} \\
i
\end{array}\right)^{2} i ! p^{i}(1-p)^{\left(\frac{3 d n}{32}-i\right)^{2}}
$$

(This expression arises from first choosing the size $i<k$ of a maximum matching $M$ between $A$ and $B$ in $R$, then choosing the vertices of $M$ in $A$ and $B$, then forming a pairing between them, then requiring all matching edges to be present in $R$, and finally requiring all edges lying outside the vertices of the matching to be absent). We can estimate the above expression from above by:

$$
\begin{aligned}
e^{-p\left(\frac{3 d n}{32}-k\right)^{2}}\left(1+\sum_{i=1}^{k}\left(\frac{3 e d n}{32 i}\right)^{2 i} i^{i} p^{i}\right) & <e^{-p\left(\frac{3 d n}{32}-\frac{d^{2} n}{32}\right)^{2}}\left(1+\sum_{i=1}^{k}\left[\left(\frac{3 e d n}{32 i}\right)^{2} i p\right]^{i}\right) \\
& <e^{-\frac{d^{2} n^{2} p}{256}}\left(1+\sum_{i=1}^{k}\left(\frac{9 e^{2} d^{2} n^{2} p}{1024 i}\right)^{i}\right) \\
& =e^{-5 k}\left(1+\sum_{i=1}^{k}\left(\frac{45 e^{2} k}{4 i}\right)^{i}\right)
\end{aligned}
$$

In the last sum each summand is easily seen to be at least twice larger than the previous summand, and hence the above estimate is at most $e^{-5 k} 2\left(\frac{45 e^{2} k}{4 k}\right)^{k}=o(1)$.

As to part (b), let $H_{0}$ consist of $\left\lfloor\frac{n}{d n+1}\right\rfloor$ disjoint cliques $C_{1}, \ldots, C_{t}$ each of size at least $d n+1$. If $H \cup R$ is $k$-connected, then each $C_{i}$ is incident to at least $k$ edges, implying $|R| \geq \frac{k t}{2}=\frac{k}{2}\left\lfloor\frac{n}{d n+1}\right\rfloor$. 
Acknowledgment: The authors thank the anonymous referees for their helpful comments.

\section{References}

[1] T. Bohman, A. Frieze and R. Martin, How many random edges make a dense graph Hamiltonian?, Random Structures and Algorithms 22 (2003), 33-42.

[2] B. Bollobás, Random Graphs, Academic Press, 1985.

[3] B. Bollobás, Random Graphs, In Combinatorics, Proceedings, Swansea 1981, London Math. Soc. Lecture Note Ser. 52, Cambridge Univ. Press, Cambridge (1981) 80-102.

[4] B. Bollobás and F. Chung, The diameter of a cycle plus a random matching, SIAM Journal on Discrete Mathematics 1 (1988) 328-333.

[5] R. Diestel, Graph Theory, Springer-Verlag, 1997.

[6] P. Erdős and A. Rényi, On the evolution of random graphs, Publ. Math. Inst. Hungar. Acad. Sci. 5 (1960) 17-61.

[7] A. Frieze, On the independence number of random graphs, Discrete Mathematics 81 (1990) 171-175.

[8] S. Janson, T. Łuczak, and A. Ruciński, Random Graphs, Wiley - Interscience Series, New York, 2000.

[9] J. Komlós and M. Simonovits, Szemerédi's Regularity Lemma and its applications in graph theory, Combinatorics, Paul Erdös is eighty, Vol. 2 (1993) 295-352.

[10] T. Euczak, The chromatic number of random graphs, Combinatorica 11 (1991) 45-54.

[11] E. Szemerédi, Regular partitions of graphs, Colloques Internationaux C.N.R.S. № 260 - Problèmes Combinatoires et Théorie des Graphes, Orsay (1976), 399-401. 